\title{
DIDÁCTICA DE LAS CIENCIAS SOCIALES Y/O DIDÁCTICA COMO CIENCIA SOCIAL
}

\begin{abstract}
Resumen
El presente documento, que hace parte de la investigación Didáctica de las Ciencias Sociales 1994-2006, recoge los debates contemporáneos sobre el estatus epistemológico de la didáctica de las ciencias sociales, DCS. Para el desarrollo de este aspecto se presentan dos perspectivas: una que ha centrado su mirada en la defensa de la didáctica como ciencia social, con un objeto de estudio definido y como una ciencia que posee las características de las ciencias sociales. La otra, que ha aportado a la didáctica de las ciencias sociales desde el qué, el cómo y a quién enseñar. Aspectos que se alimentan con la productividad académica que encontramos en el campo desde sus principales lineas de investigación, las cuales expresan los aspectos y conceptos que consolidan dicha ciencia social.
\end{abstract}

Palabras clave: Didáctica, ciencias sociales, epistemologia, didáctica de las ciencias socialos, lineas de investigación.

\section{DIDACTICS OF THE SOCIAL SCIENCES AND/OR DIDACTICS AS A SOCIAL SCIENCE}

\begin{abstract}
The present work, which does part of the investigation "Didactics of the Social Sciences 1994-2006", gathers the contemporary debates upon the epistemological status of the didactics of social sciences. DSC. For the development of this aspect, two perspectives are presented: The first one has centered their look in the defense of didactics as social science, with a definite object of study and as a science that possesses the characteristics of the social sciences. The second has contributed to the didactics of the social sciences telling what, how and whom to teach. Aspects that are fed with the academic productivity that we find in the field from its main lines of investigation, which express the aspects and concepts that consolidate such a social science.
\end{abstract}

Key words: Didactics, social sciences, epistemology, didactics of the social sciences, lines of investigation.

* Magister en Educación. Doctoranda en Estudios Latinoamericanos, UNAM, México. Profesora de la Universidad Pedagógica Nacional, Bogotá. amorales@pedagogica edu.co

** Magister en Educación. Doctoranda en Estudios Latinoamericanos, UNAM, México. Profesora de la Universidad Pedagógica Nacional, Bogotá. migonzalez@pedagogica.edu.co

Texto original recibido: 01-24-08 y aprobado: $30-04-08$ 


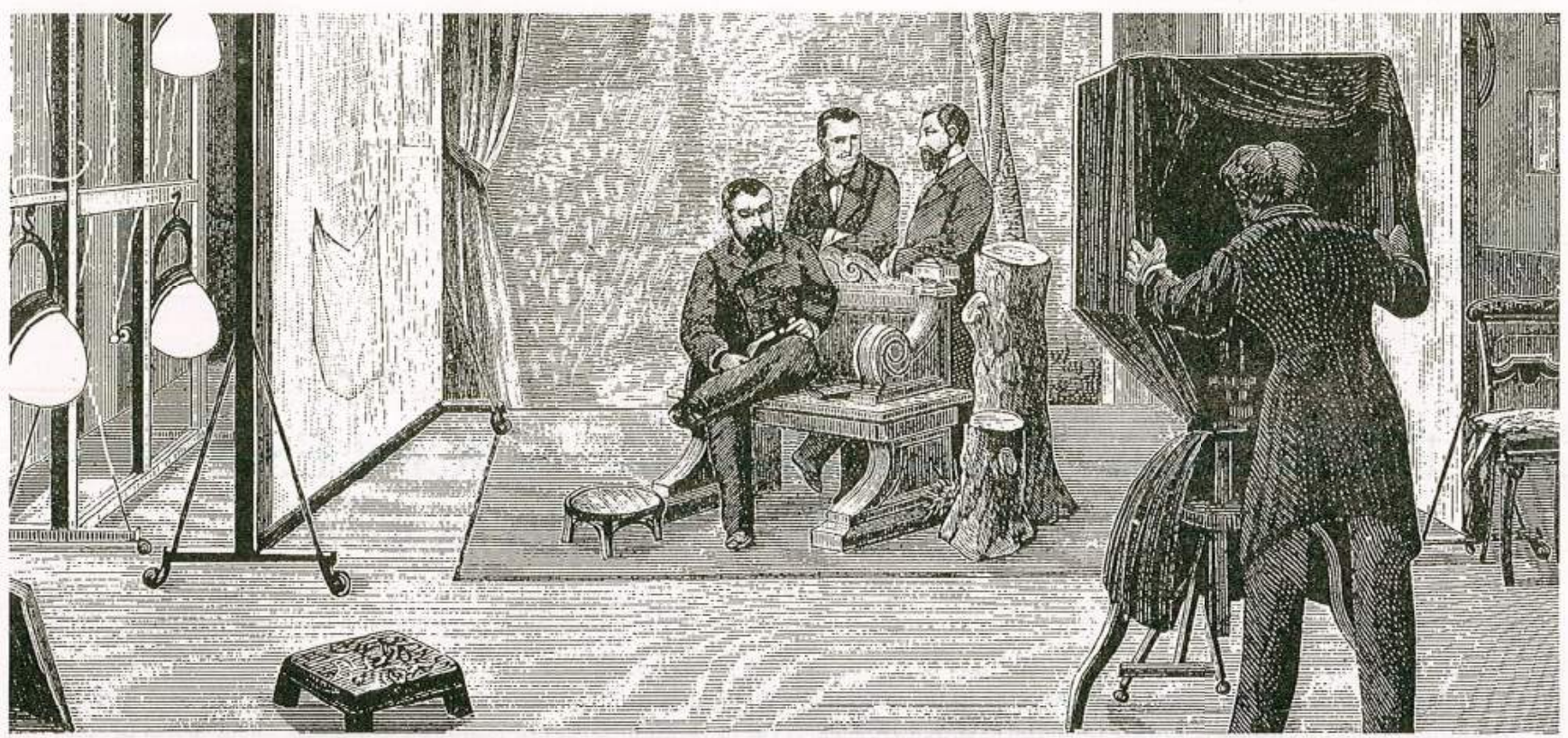

\section{INTRODUCCIÓN}

En el marco del proyecto de investigación Didáctica de las Ciencias Sociales en Educación Preescolar y Básica Primaria, que pretende hacer un balance bibliográfico de las producciones académicas del campo en el periodo 1994-2006, abordamos la articulación de dos conceptos clave que constituyen nuestro objeto de conocimiento: didáctica y ciencias sociales. Al respecto nos encontramos con reflexiones que muestran la dualidad que enuncia el título. ¿Puede hablarse de la didáctica como una ciencia social y/o didáctica de las ciencias sociales? Esta dualidad que parece confundirnos tiene más puntos de encuentro que de desencuentro.

Los dos conceptos clave que constituyen el objeto de conocimiento al cual vamos a referirnos: didáctica y ciencias sociales, denotan diferencias si se articulan desde los conectores de las ó como, los cuales dan un significado diferente a cada conjugación. Asi, hablar de didáctica de las ciencias sociales nos remite fácilmente a pensar en qué, cómo, para qué y por qué se enseñan las ciencias sociales. No obstante, hablar de didáctica como ciencia social se refiere a pensar la didáctica en términos epistemológicos.
La didáctica de las ciencias sociales, independientemente de la perspectiva, nos remite a pensar el conocimiento de lo social con sus caracteristicas: la subjetividad, la multiplicidad, la praxis, la diversidad teórica, etc.; pero al hablar de la didáctica como una disciplina social, nos obliga a pensarla como un campo de conocimiento que se inscribe dentro de las ciencias sociales, como un nuevo campo disciplinar que centra su atención en la enseñanza, diferenciándola de la didáctica de las ciencias sociales la cual nos remite a analizar cómo se ha pensado la enseñanza de las disciplinas sociales: historia, geografia, politica, economia, etc. La primera perspectiva podriamos abordarla como un campo epistemológico, en la media en que se pregunta por su objeto de estudio: la enseñanza, independiente de la disciplina a ser enseñada; la segunda perspectiva pregunta por las caracteristicas de esas disciplinas para ser enseñadas.

Podemos ubicar algunas investigaciones en ambas perspectivas: epistemológica y especifica, campos que se han venido conjugando históricamente. La didáctica como ciencia -perspectiva epistemológica- es defendida por la argentina Camilloni (1996: 35), quien afirma que al ser una ciencia social, la didáctica es un conocimiento práctico que no puede ser leido por los cánones del positivismo, sino desde una mirada critica que reconoce en la teoria práctica un conocimiento cientifico. Además, desde esta perspectiva se proponen categorías $y$ enunciados que -aunque en muchos casos son tomados de otras disciplinas sociales-, posibilitan a la didáctica interpretar y analizar la enseñanza escolar. Asi ha retomado enunciados, conceptos y análisis, sobre todo de la psicologia para ser reflexionados en la enseñanza en general, independientemente de las disciplinas escolares; por ejemplo, se toman elementos de la psicologia de la cognición social para ver los procesos de pensamiento del sujeto. Un tanto diferente sucede con la perspectiva especifica de la didáctica; para esta perspectiva, se toman elementos centrales e importantes de la disciplina a ser enseñada: sus conceptos y procedimientos, valores que son elementos clave a la hora de enseñar las disciplinas, aunque para esta mirada también son importantes los procesos pedagógicos y de aprendizaje del estudiante.

Queda claro hasta aqui que estamos hablando de dos perspectivas desde las cuales se aborda la didáctica: epistemológica y de la didáctica específica. La primera: la didáctica como ciencia social se ha nutrido de la psicologia, 
sobre todo de la línea cognitiva, y sus argumentos se basan en muchos casos en una defensa del a quién enseñar, haciendo evidente desde esta pregunta la mirada cognitiva de la didáctica. Así, algunos autores sostienen que la teoria cognitiva es fundamental si se quiere pensar en el aprendizaje del individuo y esta contribuye al campo de la didáctica de las ciencias sociales (AISEMBERG, 1994). Frente a esta, los defensores de las didácticas especificas afirman que pensar la didáctica desde la teoria cognitiva distrae del debate la compleja configuración del campo didáctico (Joaquín Prats, 1997).

Las didácticas específicas defienden la idea de que las diferentes disciplinas al ser enseñadas poseen su particularidad (no es lo mismo enseñar ciencias sociales que matemáticas, por ejemplo), y que ambas ciencias se han configurado epistemológicamente con características que las hacen únicas, por ello deben ser enseñadas desde las particularidades desde donde han sido construidas.

A pesar de esta tensión, las dos perspectivas se han nutrido reciprocamente, ya que las investigaciones que se han realizado desde las didácticas especificas han alimentado el campo de la didáctica como disciplina, y viceversa, según Maria Cristina Davini (1996). Para el caso de este trabajo defenderemos la idea de que no se esta hablando de una división de la didáctica, ni de dos campos del conocimiento que sean opuestos e irreconciliables, sino de dos perspectivas de la didáctica.

La relación entre estas dos vertientes de estudio de la didáctica se establece en que se nutren mutuamente de sus investigaciones y se busca darle un estatus como campo de conocimiento. Pero este estatus se logra con la consolidación de una producción intelectual y con la configuración de comunidades académicas que den cuenta de su objeto de estudio. Lo que encontramos es que ambos debates, que están relacionados, no suelen ser abordados en conjunto, de manera que los intelectuales y didactas buscan argumentos de orden epistémi- co para abordar esta ciencia social; y otro grupo de investigadores, maestros y didactas se centran en la producción sobre la didáctica especifica, desde ensayos, experiencias y propuestas que indican qué, cómo y a quién enseñar las ciencias sociales

\section{La didáctica como disciplina social}

La perspectiva que considera la didáctica como una disciplina social está siendo trabajada, sobre todo en Argentina, por Alicia Camilloni. Esta perspectiva plantea que al ser la didáctica una disciplina social, posee la misma complejidad de las otras. Es decir, que es un conocimiento basado en la práctica social, donde está en juego un fuerte subjetivismo, no plantea leyes universales; además, la didáctica es una disciplina prescriptiva en el sentido que pretende dar orientaciones para mejorar la práctica pedagógica. Por esta última característica, la propuesta de pensar la didáctica como una disciplina social encuentra mayores dificultades que otros conocimientos.

Respecto al carácter controvertido para su análisis epistemológico, Moore (1980) ${ }^{1}$ sostiene que la didáctica es una teoria práctica no científica por poseer un carácter prescriptivo, recomendatorio, normativo y por su estrecha relación con la actividad práctica, elementos que son incongruentes con la ciencia y por tales razones no debe ser considerada como científica. Frente a ello Camilloni defiende la idea de didáctica como ciencia social que no puede ser abordada bajo los "mismos controles que los que recibe una teoría científica" (CAMILLONI, 1996: 27), sino a partir de los supuestos teóricos con los que han sido validadas las otras disciplinas sociales. Así las cosas, se plantea que la didáctica se encuentra en el mismo nivel que otras disciplinas sociales, como la geografia, la sociologia y la historia, y debe ser analizada desde sus teorias y métodos. Sin embargo, es una disciplina de reciente aparición y adscripción a las ciencias sociales (Rodriguez, 2005: 45).

1 Citado por Camilloni. 1996, p. 26
Desde la perspectiva epistemológica varios autores asumen la didáctica como una disciplina, en la que la relación enseñanza-aprendizaje es su eje estructurante. Para Benejam la didáctica es una disciplina sintetizadora del conocimiento cientifico, la cual tiene por finalidad "cuestionar la enseñanza y el aprendizaje para ofrecer líneas de actuación que relacionen la teoria y la práctica y permitan aplicar los resultados a situaciones contextualizadas de enseñanza-aprendizaje ${ }^{\text {"2 }}$. En esta concepción de didáctica es evidente la búsqueda de un saber teórico práctico, que encuentra su utilidad al ser aplicado. Esta misma mirada la presentan Friera y Fernández (2000), para quienes la didáctica de las ciencias sociales es vista como un área del conocimiento compleja, en la que confluyen saberes de las ciencias referentes, como la historia y la geografia, junto con otras disciplinas, donde la didáctica tiene un carácter de saber aplicado, desempeñando un papel básico, el de la transposición del saber del maestro al saber del alumno. Sin embargo, los autores sostienen que la didáctica de las ciencias sociales no es un tipo de saber instrumentalizado, más bien se trata de que su función principal sea la de formar ciudadanos cultos y criticos ante su medio.

Frente a esta discusión, son importantes los aportes que se retoman de la escuela de Frankfurt con respecto al conocimiento social, puesto que desde esta se sostiene que lo social no puede ser comprendido por lógicas unánimes, donde se privilegia la razón positivista, pues la ciencia tiene estrecha relación con los problemas sociales y reales que requieren no solo su interpretación, sino la praxis para abordarlos. El nexo entre la teoria y la práctica es imprescindible (HABERMAS, 1996) para la comprensión de lo social; el conocimiento se construye a partir de esta interacción. La didáctica es una disciplina práctica al encontrarse en permanente fecundación

Citada por Trave, Gabriel. (1998). Simposium de didáctica de las ciencias sociales. Universidad de Leilla-Asociación Universitaria de Profesores, p.15. 
la teoría y la práctica como constituyentes de su discurso.

En tal sentido, la didáctica es una disciplina que se ocupa de la enseñanza en la práctica pedagógica, la cual está impregnada de valores, de posturas normativas, de intereses, etc. "La didáctica podria ser aceptada como disciplina cientifica y, en particular, como una ciencia social. Pensemos que cuenta con suficientes condiciones de validación y justificación de los conocimientos y las normas que ofrece, o podria ofrecer para la configuración de la práctica pedagógica y para la mejor comprensión de esta" (CAMILLONI, 1996).

Cuando se habla de disciplina, nos referimos a dominios de conocimiento que implican componentes como: conceptos, métodos, herramientas, pero debemos tener en cuenta que la didáctica está siendo considera como una disciplina dentro de las ciencias sociales, asi que no solo esos componentes son claves, sino el componente subjetivo y prescriptivo que únicamente podria analizarse desde una perspectiva interpretativa y crítica, no positivista del conocimiento. La didáctica apunta a desarrollar cierto tipo de intervención social; en tal sentido, es una disciplina que se pregunta por la praxis. "Si todo trabajador social se plantea cómo trabajar, cómo intervenir en comunidad, los didactas se preguntan por la enseñanza tratando de responder a estas otras preguntas complementarias y especificas: ¿Qué enseñamos? ¿Cómo lo enseñamos? ¿Qué debemos enseñar? ¿Qué debe ser y hacer la escuela?"(CAmilloni, 1996: 32). Además,

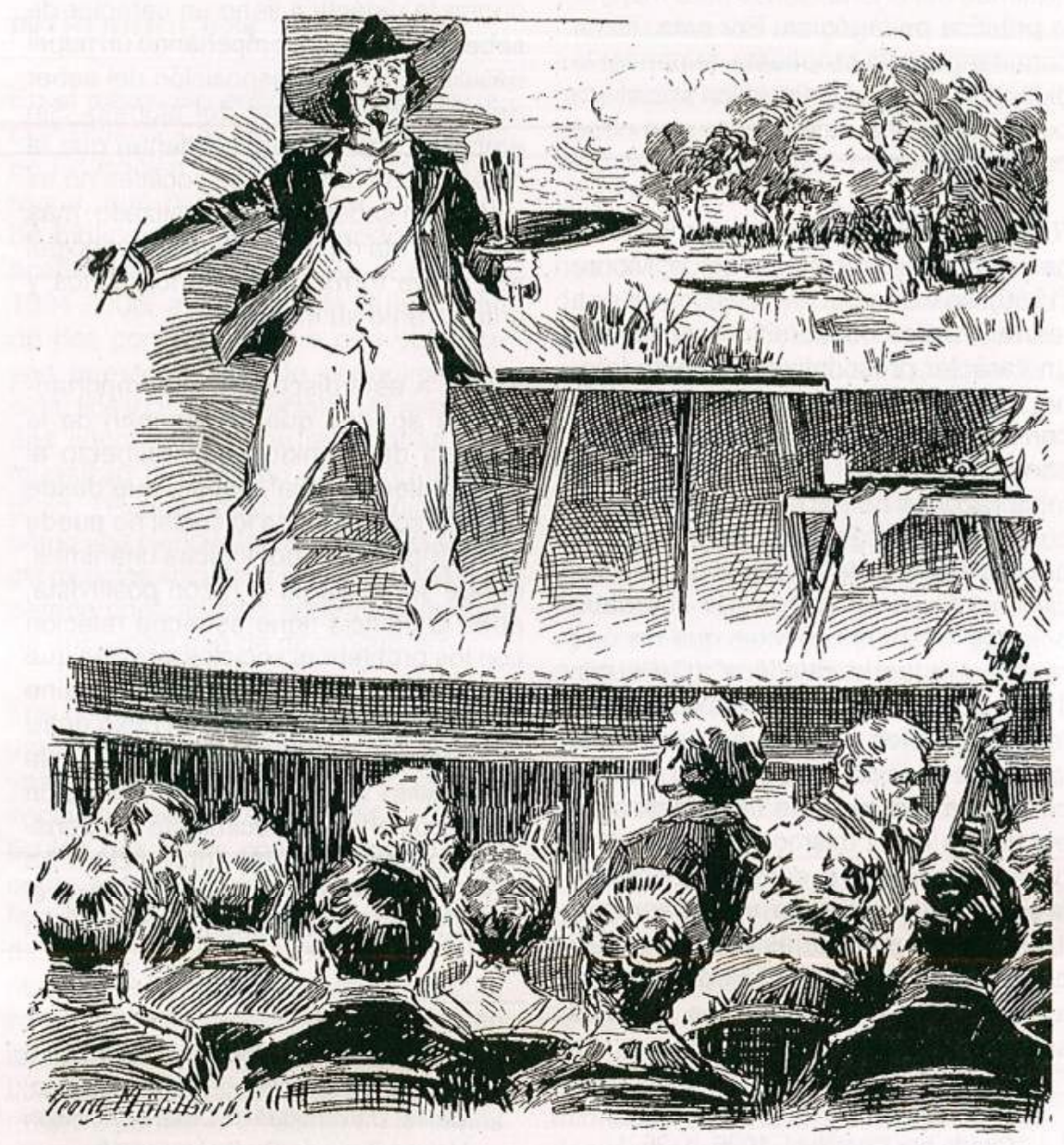

la didáctica debe ser considerada una disciplina social porque recibe de muchas de ellas aportes para configurar su campo de conocimiento que está ligado a la enseñanza. La didáctica se apoya en variadas disciplinas, como la psicologia, la sociología, la antropologia, la politica, que han venido estudiando la escuela, su curriculo, los sujetos que de ella hacen parte y han posibilitado a la didáctica, métodos, estrategias, teorias e incluso objetos de estudio.

La didáctica no solo toma de otras disciplinas, sino que las otras toman de su objeto de estudio. Al decir de Camilloni (1996:25), "los didactas, que hasta este momento eran los únicos que se ocupaban de lo que el maestro hacia en clase, encuentran que ese objeto de estudio propio es tema de interés para personas que provienen de otros campos disciplinarios, con lo cual se plantean algunos problemas".

El didacta sabe que debe utilizar los conocimientos que vienen de otros campos, no puede cerrar su mirada al análisis puramente disciplinar; además, que la didáctica como disciplina se ha constituido con el apoyo de otras múltiples ciencias, ha heredado teorias y enfoques de variadas disciplinas, y con todas ellas posee deudas. Por ello, no podria afirmarse que la didáctica posee unos limites con demarcaciones muy claras y definidas como disciplina pues por su misma configuración y las deudas que posee le posibilitan y la obligan, a entrar al terreno de otras ciencias. Aunque la didáctica le debe mucho a la psicologia, esta presenta un proceso muy diferenciado de la idea de enseñanza-aprendizaje y se centra en la enseñanza. Desde esta perspectiva la didáctica relacionada con la psicologia se interesa por la enseñanza de contenidos, y la psicologia educativa investiga sobre el aprendizaje de esos contenidos, por ello la necesaria relación entre didáctica y psicologia. Quizás esta es de las tendencias más recientes en la investigación de la didáctica en ciencias sociales en vista de la preocupación por el cómo aprenden, qué les interesa y cómo construyen el conocimiento los estudiantes. 
La didáctica, al ser una ciencia social, posee las mismas caracteristicas de las ciencias sociales en general, ya que existen diversas perspectivas que la obligan a mirar diferenciadamente al mismo objeto social; en este caso la enseñanza puede ser observada desde una mirada positivista, interpretativa y crítica. Más aún, el objeto que se construye desde esa perspectiva, a pesar de decirse común, resulta ser diferente y así mismo su interpretación sobre este: la enseñanza.

\section{La didáctica específica de las ciencias sociales}

La relación entre la disciplina didáctica y la didáctica de las ciencias sociales permite la comprensión más global de la enseñanza, pues, por un lado, obliga al docente que enseña las ciencias sociales a abordar los aportes de la didáctica como ciencia y, por otro, a la disciplina didáctica, a analizar lo que sucede en la escuela. Esta se convierte en una relación dialéctica propia del conocimiento social, no puede pensarse solo en la técnica o los instrumentos, tampoco solo en la teoria.

Ahora bien, para la didáctica de las ciencias sociales el conocimiento que le aporta la disciplina didáctica es muy importante para pensar la enseñanza del conocimiento social, pues ello posibilita reconceptualizar los contenidos, la metodologia, los objetivos, las relaciones pedagógicas en la enseñanza. Esta es una mirada muy cercana a lo que se denominan didácticas especificas en España. Se definen como campos de las respectivas ciencias por tener entre su estudio conceptos claves de las ciencias enseñadas, la particularidad de la disciplina, las teorias, su objeto de conocimiento, sus objetivos y métodos. Sobre la didáctica especifica se han planteado múltiples propuestas didácticas, que van desde la enseñanza de un tema en particular (la ciudad, por ejemplo) hasta propuestas curriculares de áreas (history 13-16 de Inglaterra).

Este aspecto puede verse reflejado en la enseñanza de la geografia y la historia. Frente a la didáctica de la historia ésta se encarga de reflexionar acerca de conceptos como el tiempo histórico, la causalidad, la empatía, los métodos de investigación histórica, las dificultades del conocimiento histórico, etc. Frente a la didáctica de la geografía se considera que se "ocupa de saberes geográficos que se enseñan en las instituciones escolares, es decir, de la teoría y la práctica de la transposición didáctica del conocimiento geográfico" (Rodriguez, 2000: 109)

En esta perspectiva existen tensiones que interrogan a la didáctica general casi siempre pensada por psicólogos - lingüistas, pues desde la didáctica especifica se afirma que el objetivo de la enseñanza de una determinada área (disciplina) es enseñarla desde sus caracteristicas, y quién más sabe de la disciplina que el que la estudió y la enseña; asi los contenidos a enseñar deben depender de profesionales de la disciplina, ya que ellos son quienes dominan el objeto de conocimiento.

Existen varias investigaciones sobre las didácticas especificas, por ejemplo, en ciencias naturales, sociales, artes, pero uno de los grupos más consolidados se encuentra en el campo de las matemáticas, con el grupo francés IREM (institutos de investigación sobre la enseñanza de las matemáticas), quienes han construido conceptos claves para su objeto de estudio, como es el caso de la transposición didáctica, que ha sido tan importante y que es retomado por otras disciplinas, convirtiéndose, al decir de los didactas generales, en conceptos claves para el análisis de la didáctica como ciencia (DAVINI, 1996: 58 ). De tal forma que la didáctica desde la perspectiva de ciencia o disciplina ha tomado unos conceptos clave de las didácticas especificas para ser analizados desde una perspectiva más generalista. Por ello resulta paradójico lo que afirma Davini (1996: 60) al decir que "la autonomización teórica y metodológica de las ciencias especiales encontraria legitimidad, si se comprobase que estas han alcanzado la producción de marcos conceptuales propios, de existencia y significación especifica en el marco de cada microdisciplina". Pero lo que se ha demostrado según su mismo articulo es que las didácticas generales toman conceptos construidos por las didácticas especificas.

Como se ha mostrado en algunos ejemplos, las didácticas específicas, lejos de ser una corriente contraria y de tensión con la didáctica como ciencia, ellas la han alimentado y han contribuido a configurar su campo de conocimiento, pues varios de los "postulados" de la didáctica general han sido planteados desde las especificas, y viceversa; sin embargo, sí poseen intereses diferenciados.

La didáctica de las ciencias sociales como un campo conceptual es de reciente aparición, es un campo que esta siendo estudiado desde algunos colectivos e investigadores en Iberoamérica; es el caso del grupo de la revista GRAO de España, quienes sistemáticamente vienen aportando en este campo; las publicaciones argentinas entre las que se encuentran las de Silvia Alderoqui, Silvia Finocchio, Beatriz Aisemberg, Gustavo laies, Grupo Giron. De igual manera se encuentran las investigaciones adelantadas por Gabriel Travé (1998) y Joaquín Prats $(1997)^{3}$. Para el desarrollo de este campo conceptual el debate epistemoló-

3 En el ámbito internacional las investigaciones sobre la didáctica de las ciencias sociales parten de la revisión y balance de la producción académica al respecto, y son concluyentes al presentar que este es un ámbito de producción incipiente. Al respecto se puede consultar: ARraez Martinez, Juan Miguel. (2000). Fundamentos didácticos de las áreas curriculares. Madrid: Sintesis: Garcia, Antonio Luis. (1996). Principales líneas de investigación en Didáctica de las Ciencias Sociales. En El Saber en el espacio didáctico. Luis A. Ruiz Higueras (Editora). Jaén: Universidad de Jaén; Liceras Ruiz, Ángel. (1996). Didáctica de las Ciencias Sociales y Psicologia Educativa. En El Saber en el espacio didáctico. Luis A. Ruiz Higueras (Editora). Jaén: Universidad de Jaén; Travé González Gabriel. (1998). La investigación en didáctica de las Ciencias Sociales. Perspectivas y aportaciones desde la enseñanza y el aprendizaje de las nociones económicas. Huelva: Universidad de Huelva. 
gico ha sido uno de sus pilares, ya que es a partir de este que se ha buscado darle un sentido y una orientación clara a la didáctica de las ciencias sociales como disciplina científica.

Al analizar los aspectos sobre los que gira la investigación en didáctica de las ciencias sociales, sobre qué conceptos se ha constituido esta, elementos como el curriculo, el docente, el estudiante y otros conceptos emergentes 0 , en menor grado, de desarrollo han sido componentes que han contribuido al desarrollo de la didáctica especifica. Al respecto encontramos, a partir de la consulta transversal de algunos textos ${ }^{4}$ sobre la didáctica de las ciencias sociales, que seis lineas de investigación son las que más se han trabajado en el campo; éstas las presentamos a continuación de acuerdo con el grado de desarrollo, consolidación y mención que hace la bibliografia consultada.

\section{Didáctica y currículo de ciencias sociales}

Aqui se encuentran investigaciones referidas al diseño y desarrollo curricular, dedicadas a diagnosticar las situaciones y los problemas en el aula a partir de la evaluación de propuestas y materiales didácticos. La linea de investigación sobre currículo aparece en la mayoría de los documentos que presentan un estu-

4 Los siguientes trabajos hacen mención a dicha linea de investigación: ARRAÉz MARTiNez, Juan Miguel. (2000). Fundamentos didácticos de las áreas curriculares. Madrid: Sintesis; Garcia, Antonio Luís. (1996). Principales lineas de investigación en Didáctica de las Ciencias Sociales. En El Saber en el espacio didáctico. Luis A. Ruiz Higueras (Editora). Jaén: Universidad de Jaén; Liceras Ruiz, Ángel. (1996). Didáctica de las ciencias sociales y psicologia educativa. En El Saber en el espacio didáctico. Luis A. Ruiz Higueras (Editora). Jaén: Universidad de Jaén; Travé González Gabriel. (1998). La investigación en didáctica de las Ciencias Sociales. Perspectivas $y$ aportaciones desde la enseñanza y el aprendizaje de las nociones económicas. Huelva: Universidad de Huelva. dio sobre las tendencias o lineas de investigación en didáctica de las ciencias sociales; ello indica que es de las más fuertes y estructuradas, que goza de reconocimiento dentro de la comunidad académica. Desde el currículo también se estudia la presencia de la geografia. la historia y demás ciencias sociales, en los diversos curriculos escolares, a lo largo del tiempo y en los distintos niveles educativos.

En la lectura de esta línea, más que evidenciarse debates sobre la función social del curriculo de ciencias sociales, se encuentra un grupo mayoritario que se centra en aspectos del curriculo como: propuesta de diseños y desarrollos, funcionamiento, implicaciones en el aula, orientaciones didácticas y elaboración de materiales curriculares para aplicarlos, el análisis de contenidos, el papel de los libros de texto, la identificación y diagnóstico de la situación, problemas particulares del aula, los métodos, contenidos y fundamentación de las áreas sociales, y la evaluación de los mismos.

\section{El docente en la enseñanza \\ de las ciencias sociales}

Una segunda línea de investigación que tiene consenso es la referida al lugar del docente. Esta linea de investigación agrupa varias tendencias: en unas se centra la mirada sobre la formación inicial del docente, desde sus concepciones, hábitos, conocimientos y desarrollo profesional. Otra, busca-desde las concepciones y pensamiento del profesor-. analizar para ayudar a comprender cómo construye su realidad educativa, cómo inciden estos aspectos en el desarrollo de su propia práctica.

Igualmente, pueden mencionarse tendencias investigativas en las que se asume al maestro como sujeto de enseñanza, quien se encarga de adecuar contenidos, materiales y estrategias de enseñanzaaprendizaje, en manos de quién está el estancamiento y/o cambio educativo, es decir, la innovación educativa.

Algunos ejes que estructuran esta línea de investigación son: formación pro- fesional; vocación, interés, capacidad intelectual; crecncias e ideas sobre educación, enseñanza y ciencias sociales; conocimiento de la disciplina conocimiento pedagógico de la materia; Cambio de concepciones sobre enseñanza: incertidumbres de la práctica, entre otras.

\section{El estudiante en las ciencias sociales}

Varias de las investigaciones en didáctica de las ciencias sociales ubican al estudiante como la razón de ser de la enseñanza, por ello se estima que este debe ser tenido en cuenta desde sus intereses, motivaciones, niveles de desarrollo, dificultades de aprendizaje y demás aspectos cognitivos. En estas investigaciones priman los enfoques de la psicología en sus diferentes especificidades: evolutiva, educativa, cognitiva. Se destacan trabajos sobre el grado de motivación e interés por la materia; los conocimientos previos generales e instrumentales sobre el área; teorias implicitas acerca de los temas específicos: conocimientos 0 aprendizajes propios de cada etapa de desarrollo; valores y actitudes que practican, etc.

Tal como lo menciona Travè (1998), las investigaciones referidas al alumno provienen en su mayoria del campo de la psicología educativa, preocupada por la descripción del tipo de representaciones de los alumnos en la construcción del conocimiento y por las dificultades implicitas en este proceso de elaboración de esquemas mentales 5 .

5 Ampliando esta idea, Liceras Ruiz, Ángel. (1996: 66-67), condensa las aportaciones de la psicologia a la didáctica en cuatro aspectos, que se refieren al estudiante y su relación con el proceso de aprendizaje: 1. Las investigaciones realizadas por Plaget y sus colaboradores relativas al aprendizaje del espacio, el tiempo, la causalidad, el juicio moral, etc., en relación con las etapas del desarrollo psicomotor de los sujetos. 2. Los trabajos sobre psicologia cognitiva en relación con los aspectos del aprendizaje del ámbito de las ciencias sociales llevados a cabo por Asensio; Carretero Delval; Pozo; Marchesi; Ochaita; Martín 


\section{Conceptos estructurantes en la didáctica de las ciencias sociales}

Esta línea de investigación se encuentra menos referenciada en la literatura especializada, en relación con las anteriores $^{6}$, ligadas a los procesos cognitivos sobre el aprendizaje de las ciencias sociales $y_{\text {, en concreto, a variables }}$ como: espacio, tiempo, explicaciones causales, representaciones sociales, formación de conceptos, etc. Aquí pueden mencionarse investigaciones referidas a la formulación de propuestas, orientaciones didácticas, elaboración de materiales curriculares para aplicarlos en la enseñanza de las ciencias sociales, en las diferentes etapas del sistema educativo.

\section{El patrimonio artístico, ambiental, ecológico y las nuevas tecnologías}

Estas son líneas que podriamos denominar actuales, pues en términos de las problemáticas mundiales frente al uso, conservación, respeto y preservación del medio ambiente, se empieza a indagar y construir una linea investigativa que recoge trabajos sobre la didáctica del medio ambiente $\mathrm{e}$ investigaciones de orden ecológico, centradas en lo procesos de enseñanza-aprendizaje en su totalidad; es decir; que describen dichos procesos a partir de un análisis multidisciplinar ${ }^{7}$. También se encuentran investigaciones sobre la didáctica del patrimonio cultural y medioambiental, que permiten estudiar las posibilidades didácticas del patrimonio, su función

etc. 3. Las aportaciones de psicólogos como: Dewey; Yaba; Bruner; Blom; Gagné y Nova, en relación con diversos aspectos de la didáctica de las ciencias sociales. 4. Las contribuciones de Coll y su equipo (Mauri; Solé; Del Carmen: Zabalza, etc.) en cuanto al desarrollo de las teorias cognitivas en la educación.

6 Al respecto solo es citada por Friera y Fernández (2000); Prats (1997); y Garcia, Antonio Luis (1996)

7 Esta línea de investigación es presentada en varios trabajos, se sugiere revisar Friera y Fernández (2000); Gabriel Travé González (1998); y Prats (1997). social, educativa e ideológica (PrATs, 1997: 23).

En menor medida se mencionan inves- tigaciones relacionadas con aspectos como el patrimonio artístico, planteamientos interdisciplinarios con otras áreas curriculares, el uso de la prensa en ciencias sociales. Igualmente, aparecen investigaciones relacionadas con el uso de las nuevas tecnologías en el proceso de enseñanza-aprendizaje de las ciencias sociales (Friera y Fernández, 2000). Varios de estos aspectos se trabajan desde una mirada interdisciplinaria, ya que posibilita integrar contenidos y diferentes perspectivas sobre las nuevas tecnologias

Lineas de investigación emergentes o con menor grado de desarrollo

En esta línea de investigación se agrupan las mencionadas por varios autores pero que no presentan consenso entre ellos, y que en la mayoria de los casos se mencionan de manera aislada. De esta mancra se evidencian campos de investigación que no se han consolidado, que están emergiendo e incluso que han sido abandonados. Entre ellas encontramos:

- Influencia de los contextos en los procesos de aprendizaje de los estudiantes.

- Evaluación en ciencias sociales: función, criterios, técnicas e instrumentos.

- Carácter histórico que se refiere al proceso formativo de la enseñanza de las Ciencias sociales en los planes de estudio del maestro.

- Materiales curriculares: libros de texto utilizados en diferentes momentos históricos

Este es el panorama sobre la investigación en didáctica desde los textos de didáclica de las ciencias sociales que llegan al contexto colombiano. Ahora bien, las investigaciones realizadas en el ámbito nacional son incipientes. Muestra de ello son la poca investigación, producción y difusión sobre la temática, el reducido número de grupos de investigación especializados en este campo y la emergencia de más temas y/o cátedras transversales como: constitución politica y democracia, afrocolombianidad, ética y derechos humanos, entre otras, que demandan a las ciencias sociales escolares trabajar estos ámbitos formativos, fragmentando cada vez más la labor en la enseñanza y el aprendizaje de las ciencias sociales.

Este primer acercamiento a los debates y producciones en torno a la didáctica en las ciencias sociales nos deja algunos problemas para seguir abordando: ¿En qué grado de desarrollo se encuentra la didáctica como ciencia social en nuestro país? ¿Qué tipo de producción académica es la que circula e influye en nuestro contexto? ¿En qué grado de desarrollo se encuentra la producción en el tema cn el ámbito nacional respecto al internacional?

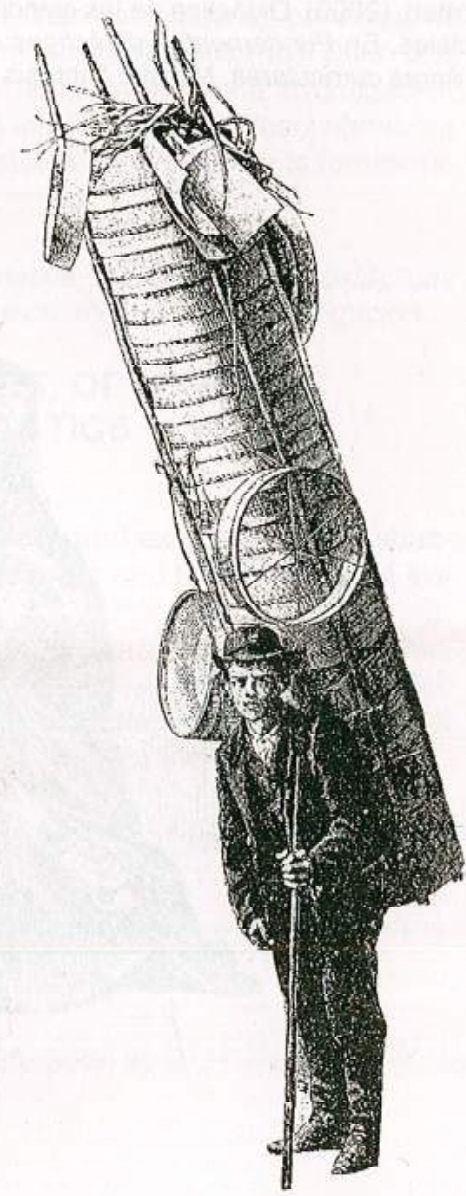




\section{BIBLIOGRAFIA}

AISENBERG, Beatriz. (1994). Por qué y cómo trabajar en el aula con los conocimientos previos de los alumnos: un aporte de la psicologia genética a la didáctica de los estudios sociales para la escuela primaria. En Didáctica de las ciencias sociales. Buenos Aires: Paidós.

Arraez Martinez, Juan Miguel. (2000). Fundamentos didácticos de las áreas curriculares. Madrid: Sintesis.

Camilloni, Alicia. (1996). De herencias, deudas y legados. Una introducción a las corrientes actuales de la didáctica. En Corrientes didácticas contemporáneas. Buenos Aires: Paidós.

DaviNı, Maria Cristina. (1996). Conflictos en la evolución de la didáctica. La demarcación de la didáctica general y las didácticas generales. En Corrientes didácticas contemporáneas. Buenos Aires: Paidós.

Friera SuÁrez, Florencio, Fernández Rubio, Carmen. (2000). Didáctica de las ciencias Sociales. En Fundamentos didácticos de las áreas curriculares. Madrid: Sintesis.
Garcia, Antonio Luis. (1996). Principales líneas de investigación en Didáctica de las Ciencias Sociales. En El Saber en el espacio didáctico. Luís A. Ruiz Higueras (editora) Jaén: Universidad de Jaén.

HABERMAS, Jürgen. (1996). Teoria y praxis. Buenos Aires: Ariel.

IAIES, Gustavo y Segal, Analia. (1994). La escuela primaria y las ciencias sociales: una mirada hacia atrás y hacia delante. En Didáctica de las ciencias sociales. Buenos Aires: Paidós.

Liceras Ruiz, Ángel. (1996). Didáctica de las Ciencias Sociales y Psicologia Educativa. En El Saber en el espacio didáctico. Luis A. Liceras Ruiz (editor). Jaén: Universidad de Jaén.

MOORE, T. (1980). Introducción a la teoría de la educación. Madrid: Alianza.

Prats, Joaquín. (1997). Los caminos de la didáctica de las ciencias sociales. Grupo IBER. Revista Grao. España.
Rodriguez, Francisco. (2000, abril). La elaboración del conocimiento escolar: ¿de la ciencia geográfica a la geografía que se enseña? En Serie IBER. Didáctica de las ciencias sociales geografia e historia. Revista Grao, 24

Rodriguez Ratia, Federico. (2005). En Dominguez Garrido, Maria (coord.). Didáctica de las ciencias sociales. Pearson Prentice Hall.

Travé GonzAlezz. Gabriel. (1998). La investigación en didáctica de las Ciencias Sociales. Perspectivas y aportaciones desde la enseñanza y el aprendizaje de las nociones económicas. Huelva: Universidad de Huelva.

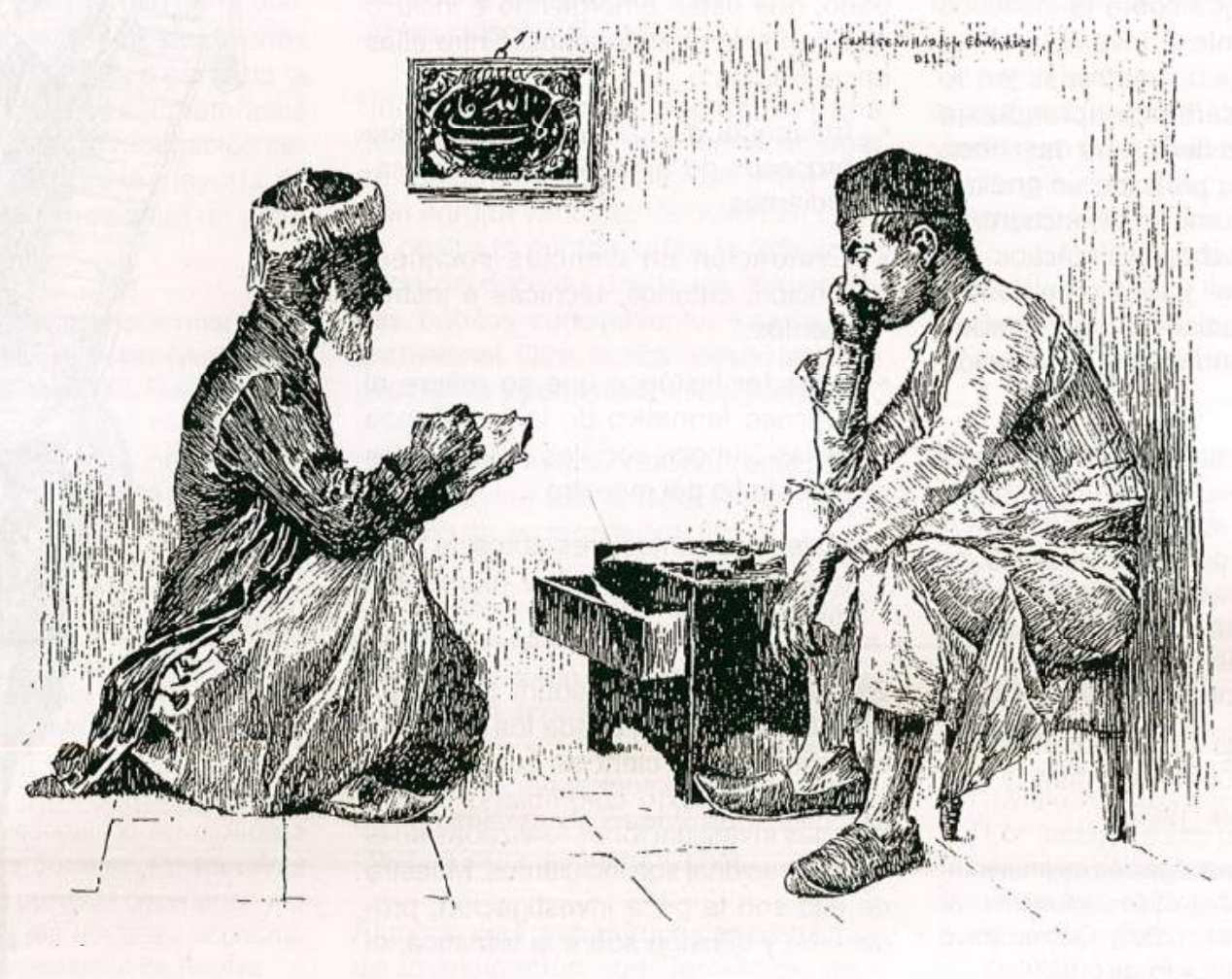

